\title{
Effectiveness of Epidural Analgesia, Continuous Surgical Site Analgesia, and Patient-Controlled Analgesic Morphine for Postoperative Pain Management and Hyperalgesia, Rehabilitation, and Health-Related Quality of Life After Open Nephrectomy: A Prospective, Randomized, Controlled Study
}

Xavier Capdevila, MD, PhD,* Sebastien Moulard, MD,* Christian Plasse, MD,* Jean-Luc Peshaud, MD,* Nicolas Molinari, PhD, † Christophe Dadure, MD, PhD,* and Sophie Bringuier, PharmD, PhD*

\begin{abstract}
BACKGROUND: There is no widely recognized effective technique to optimally reduce pain scores and prevent persistent postoperative pain after nephrectomy. We compared continuous surgical site analgesia (CSSA), epidural analgesia (EA), and a control group (patient-controlled analgesic morphine) in patients undergoing open nephrectomy.

METHODS: Sixty consecutive patients were randomized to be part of EA, CSSA, or control groups postoperatively for 72 hours. All patients received patient-controlled analgesic morphine, if needed. Hyperalgesia was assessed on the first, second, and third postoperative days. Chronic pain characteristics and quality of life were analyzed at 1 and 3 months. The primary outcome was the pain score at 24 hours. Secondary outcomes were morphine consumption, postoperative rehabilitation, hyperalgesia, chronic pain incidence, and quality-of-life parameters. RESULTS: At 24 hours, mean \pm standard deviation pain values at rest $(2.4 \pm 1.7,2.2 \pm 1.2$, and $4.2 \pm 1.2$, respectively, in EA, CSSA, and control groups, $P<.001$ ) and during coughing was lower in the EA and CSSA groups. Total morphine consumption was higher in the control group. Rehabilitation parameters improved sooner in the EA and CSSA groups. Median values of area of hyperalgesia differed at 48 hours between the EA group and the control group $\left(36.4 \mathrm{~cm}^{2}\right)$ and $\left(52 \mathrm{~cm}^{2}\right)(P=.01)$ and at 72 hours among the EA group, CSSA group, and the control group (40 $\mathrm{cm}^{2}, 39.5 \mathrm{~cm}^{2}$, and $59 \mathrm{~cm}^{2}$, respectively; $\left.P=.002\right)$. CSSA reduced the severity of pain and hyperalgesia at 1 month and optimized quality of life 3 months after surgery (role physical scores, $P=.005$ ).

CONCLUSIONS: CSSA and EA significantly improve postoperative analgesia, reduce postoperative morphine consumption, area of wound hyperalgesia, and accelerate patient rehabilitation after open nephrectomy. CSSA significantly reduces the severity of residual pain 1 month after surgery and optimizes quality-of-life parameters 3 months after surgery. (Anesth Analg 2017;124:336-45)
\end{abstract}

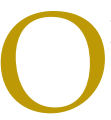
pen surgery is still a common approach for radical or partial nephrectomy. ${ }^{1,2}$ Open nephrectomy is considered to lead to intense postoperative pain $^{3}$ and a high incidence of chronic postoperative pain. ${ }^{4}$ Optimal management of postoperative pain and recovery

From the *Departments of Anesthesiology and Critical Care Medicine and Inserm Unit 1051 INM, Montpellier University Hospital and University Montpellier, Montpellier, France; †PhyMedExp, University of Montpellier, Montpellier, France; and $\ddagger$ Department of Medical Statistics, Montpellier University Hospital, Montpellier, France.

Accepted for publication September 16, 2016.

Funding: Institutional.

The authors declare no conflicts of interest.

Reprints will not be available from the authors.

Address correspondence to Xavier Capdevila, MD, PhD, Department of Anesthesiology and Critical Care Medicine and Inserm U 1051, INM and Lapeyronie University Hospital, Route de Ganges, 34295 Montpellier Cedex 5, France. Address e-mail to x-capdevila@chu-montpellier.fr. is an important challenge after major surgery. Multimodal analgesia is now recommended to decrease postoperative pain and reduce adverse drug effects. Epidural analgesia (EA) is widely recognized as an effective technique after open nephrectomy ${ }^{5-8}$ to reduce pain scores and the need for analgesics and to prevent persistent postoperative pain. ${ }^{9}$ EA was also found to reduce surgical stress responses and improve both functional capacity and health-related quality of life (HRQL). ${ }^{10}$ Although it is recognized that EA prevents postoperative pain, this technique is not systematically used as the standard of care as a result of incidences of postoperative adverse events. ${ }^{6,7}$ Continuous wound infiltration or continuous surgical site analgesia (CSSA) is an interesting alternative to EA within multimodal postoperative pain management. ${ }^{11}$ After open nephrectomy, this technique has been shown to improve pain relief, reduce opioid consumption, and accelerate recovery and discharge. ${ }^{12-14}$ These 2 analgesic techniques provided effective analgesia as part of a multimodal approach after major abdominal surgery or open nephrectomy. However, the evaluations were only placebo-controlled trials. Recently, the benefits of 
EA compared with CSSA have been challenged in patients undergoing elective open colorectal surgery. ${ }^{15,16}$ Jouve et $\mathrm{al}^{15}$ showed some advantages of EA in improving postoperative pain management and functional recovery, and Bertoglio et $\mathrm{al}^{16}$ emphasized the use of CSSA in the same context. More studies comparing EA and CSSA and including a controlled patient group are needed to evaluate the short- and longterm impact of these regimens after upper abdominal surgery and open nephrectomy. We hypothesized that CSSA may improve postoperative pain, postoperative HRQL, and the pain relief/adverse events ratio compared with EA and IV morphine patient-controlled analgesia (PCA). The primary outcome was the pain score at 24 hours. Secondary outcomes were morphine consumption, postoperative rehabilitation, hyperalgesia, chronic pain incidence, and qualityof-life parameters.

\section{METHODS}

This prospective, randomized, controlled study was approved by the Institutional Committee for the Protection of Human Subjects in Biomedical Research (Comité de Protection des Personnes, Sud-Méditerranée III, Nimes, France, No. 20100101), registered (Trial registration: Eudra CT number 2009-A01272-55; CCTIRS: 12.660), and written informed consent was obtained for all patients.

\section{Study Population}

Sixty consecutive adult patients scheduled for open total or partial nephrectomy for cancer via lumbotomic access were screened and enrolled between March 2011 and June 2012. Inclusion criteria were age 18 years and older; American Society of Anesthesiologists physical status I, II, or III; cognitive capacity to use the visual analogic scale (VAS); ability to answer the HRQL; and the chronic pain questionnaires. Medical exclusion criteria were coagulation disorders; consumption of antiaggregant or anticoagulant drugs in the last 7 days; hepatic or renal insufficiency; allergy to local anesthetic; obesity (body mass index $>30 \mathrm{~kg} / \mathrm{m}^{2}$ ); chronic preoperative consumption of opioids; psychiatric disorders; and inability to use a PCA device.

\section{Perioperative Care}

All surgical procedures were performed by senior surgeons. Patients fasted preoperatively. Patients were premedicated with $5 \mathrm{mg}$ oral midazolam 1 hour before induction of anesthesia. Standard monitoring included continuous recording of the heart rate and electrocardiography, automatic blood pressure, pulse oximetry, end-tidal carbon dioxide concentration, and esophageal body temperature. Anesthesia was standardized for all patients in the study. Induction was performed with propofol $(2.5-5 \mathrm{mg} / \mathrm{kg})$, sufentanil $(0.2 \mu \mathrm{g} / \mathrm{kg})$, cisatracurium $(0.15 \mathrm{mg} / \mathrm{kg})$, and maintenance of anesthesia was accomplished with desflurane (maximal allowable concentration $0.6 \%-1 \%)$, sufentanil $(0.3 \mu \mathrm{g} / \mathrm{kg} / \mathrm{h})$ to target a bispectral index (BIS Technology; Aspect Medical Systems, Meern, The Netherlands) between 40 and 50. Muscle paralysis was maintained by continuous infusion of cisatracurium $(0.2 \mathrm{mg} / \mathrm{kg} / \mathrm{h})$ as indicated by orbicular nerve stimulation (train of 4). After tracheal intubation, mechanical ventilation of the patients was ensured with a tidal volume of $8 \mathrm{~mL} /$ $\mathrm{kg}$, a positive end-expiratory pressure of $6 \mathrm{~cm} \mathrm{H}_{2} \mathrm{O}$, and an inspired oxygen fraction of $50 \%$ in air. All patients had urinary catheters. Normothermia was maintained using a pulsed warm air system and prophylactic antibiotherapy was given as recommended.

\section{Study Protocol}

Study information was explained to the patients by the anesthesiologists 3 weeks before surgery and at the preanesthetic consultation. Patients who consented to the project were stratified for eligibility in the study the day before surgery and baseline variables were evaluated. On the morning of the surgical procedure, the patients were randomly assigned to 1 of 3 groups using a computer-generated randomization table: EA group, CSSA group, or control group (Figure 1). For both ethical and practical reasons, according to the Patient Protection Committee request, the control group was not a placebo group and patients were nonblinded. Both the patient and the investigator were not blinded at least up to the first 72 postoperative hours. In the EA group, an epidural catheter was inserted before induction of anesthesia in a preinduction room. The procedure was performed in a sitting position. The needle entry point was situated between T7 and T8 vertebrae. An 18-G, $100-\mathrm{mm}$ Tuohy needle was used and 3 to $5 \mathrm{~cm}$ of a $20-\mathrm{G}$ epidural catheter was inserted into the epidural space. Two milliliters of a test dose solution of $2 \%$ lidocaine with epinephrine was administered. Any sensory block (cold swab test) was noted 20 minutes after the procedure. We waited 20 minutes after the test dose to rule out intravascular injection or subarachnoid or subdural block. Thirty minutes before the end of the surgery, patients received a bolus of 10 $\mathrm{mL}$ of $0.2 \%$ ropivacaine followed by a continuous infusion at $10 \mathrm{~mL} / \mathrm{h}$ for 72 hours. In the CSSA group, 2 multiholed catheters (Painfusor; Baxter Company, Maurepas, France) were inserted by the surgeon at the end of surgery using the technique previously reported by Beaussier. ${ }^{11}$ The first catheter was positioned after the closure of transverse muscle, between the transverse and internal oblique muscles. Internal and external oblique muscles were subsequently closed. The second catheter was inserted in the subcutaneous space. The subcutaneous space and the skin were closed separately. A transparent dressing covered both the catheters. Five milliliters of $0.2 \%$ ropivacaine were administered followed by continuous infusion at $5 \mathrm{~mL} / \mathrm{h}$ in both catheters using elastomeric pumps for 72 hours. The control group received a multimodal analgesia regimen with paracetamol, nefopam, and PCA morphine. For all patients, $1 \mathrm{~g}$ paracetamol and $20 \mathrm{mg}$ nefopam were administered 30 minutes before the skin was closed. Antiemetic drugs (8 $\mathrm{mg}$ dexamethasone sodium phosphate) were administered 10 minutes before the end of surgery.

\section{Postoperative Care}

After tracheal extubation and discharge from the operative room, patients were admitted to the postanesthesia care unit (PACU). All patients were titrated using $2 \mathrm{mg}$ morphine bolus every 5 minutes to obtain a pain VAS value $<4$. The postoperative PCA pump infusion (Alaris Medical System, Hampshire, UK) was started to deliver $1 \mathrm{mg}$ morphine and 


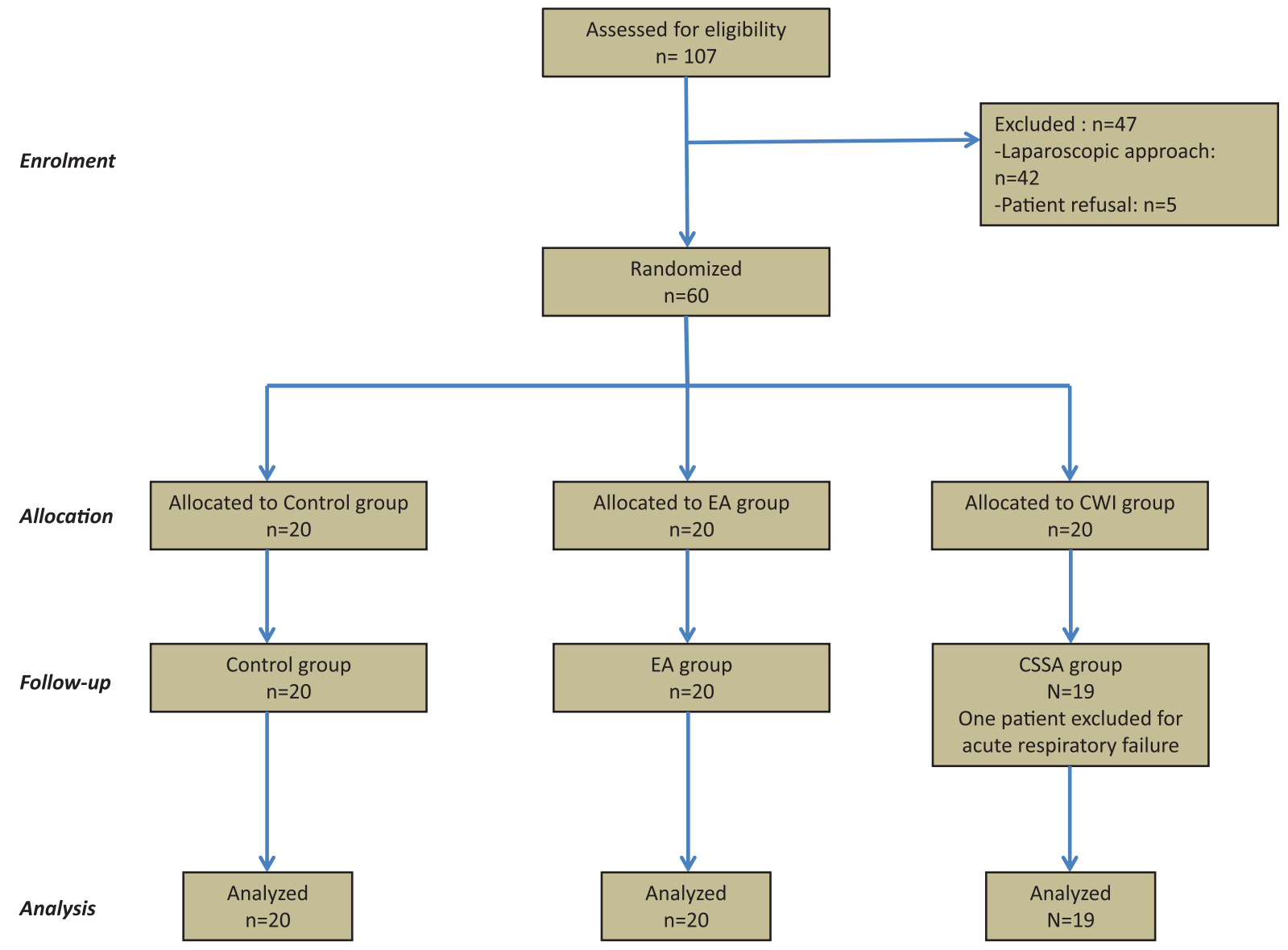

Figure 1. Flow diagram of patients in the study.

$0.05 \mathrm{mg} / \mathrm{mL}$ droperidol on demand with a 7-minute lockout time and a maximal of $15 \mathrm{mg} / 4 \mathrm{~h}$. All patients were asked to use a bolus of IV morphine if needed as pain medication. Epidural and multiholed wound catheters were removed from patients 72 hours after surgery. Nausea and vomiting were treated with $4 \mathrm{mg}$ ondansetron IV as first-line therapy and $1.25 \mathrm{mg}$ droperidol as rescue therapy. All patients were given $1 \mathrm{~g}$ paracetamol IV and $20 \mathrm{mg}$ nefopam IV every 6 hours. All patients were initially hospitalized in a specific continuous care unit (CCU) until they satisfied 4 conditions: pain VAS at rest $<4$; weaning from oxygen by nasal cannula based on oxygen saturation $>94 \%$; bowel recovery (defined by bowel movements and flatus); and time out of bed (more than 2 hours chairside). The time to return for each parameter and the duration of CCU stay for the patients in the 3 groups were noted. Postoperative hypotension, defined as a mean arterial pressure $\leq 60 \mathrm{~mm} \mathrm{Hg}$, was treated with lactated Ringer's fluid and/or vasopressors, as appropriate. Pain at rest was assessed using a 10-cm VAS at 1, 6, 12, 24, 36,48 , and 72 hours after arrival in the recovery room and pain during coughing from 24 to 72 hours. The daily total consumption of morphine was evaluated before leaving the PACU at 24, 48, and 72 hours after surgery. Urinary catheters were removed on the day after surgery. The postoperative area of hyperalgesia for punctate mechanical stimuli around the surgical incision was assessed as previously reported ${ }^{9}$ everyday for 72 hours using a Semmes-Weinstein $10 \mathrm{~g}$ (size
5.07) monofilament (Bioseb; In Vivo Research Instruments, Vitrolles, France). With the patient's eyes closed, the tests were done along radial lines from the pain-free skin area to the surgical incision at $0.5-\mathrm{cm}$ intervals until pain sensation was reported by the patient (painful, burning, sharp). The measurement was repeated by increments of $5 \mathrm{~cm}$ around the surgical incision. The measures were reported on a graph paper to calculate the total area of hyperalgesia. The occurrence of side effects in the 3 groups (eg, nausea, vomiting, acute respiratory failure, pruritus, urinary retention, local vein inflammation, fever, hypotension, cardiovascular problems, problems with catheters, local anesthetic toxicity signs, paresthesia, sedation) were recorded until discharge. All the parameters (pain VAS values, morphine consumption, rehabilitation, hyperalgesia, side effects) in the postoperative period were recorded by 2 research physicians not involved in the primary phase of the study and intraoperative patient care. The incidence of postoperative residual pain or discomfort was evaluated at 1 and 3 months after surgery using a previously published questionnaire with selected items. ${ }^{9}$ The area of hyperalgesia for punctate mechanical stimuli around the surgical incision was not assessed using Semmes-Weinstein 10-g (size 5.07) monofilaments. The patient was asked to describe the frequency and the intensity of pain, its localization, impact on activities of daily living, and the need for analgesics. HRQL was assessed preoperatively at 1 and 3 months postoperatively 
using the SF-36 questionnaire. ${ }^{17}$ This 36 -item scale is used to assess several domains. Four subscales (physical functioning [PF]; role physical [RP]; bodily pain [BP]; general health) contribute to the physical component score (PCS) and 4 subscales (vitality [VT]; mental health [MH]; role emotional [RE]; social functioning [SF]) contribute to the mental component score (MCS). The questionnaires were then sent back to the hospital by the patients.

\section{Statistical Analysis}

Categorical data were expressed as absolute value (n) or frequencies (\%). Continuous data were expressed as mean \pm standard deviation (SD) or median [interquartile range] and the Shapiro-Wilk test was used to test the normality of the distribution.

Comparisons of categorical data among groups were performed by the $\chi^{2}$ test or the Fisher exact test, as appropriate. Continuous data comparisons among groups were performed with analysis of variance or a nonparametric Kruskal-Wallis test in case of too small size or non-Gaussian distribution of the data. When a global significant difference appeared, a $2 \times 2$ two comparison was performed with the appropriate test (Student or Wilcoxon test for quantitative variables; $\chi^{2}$ test or the Fisher exact test for categorical variables).

The comparisons of paired variables (change in residual pain, daily impact... at home in all patients or for each group separately) were performed using the Student or Wilcoxon paired-sample test for quantitative variables and the McNemar test for categorical variables.

A value of $P<.05$ was considered significant. For multiple testing, we assumed $P<.01$ as significant for the post hoc analyses.

Linear mixed models were used to analyze the longitudinal data for SF-36 subscales. We used fixed effects for preoperative score, group (EA, CSSA, control), postoperative time $(1,3$ months), and their interaction (group $\times$ postoperative time). We used patient as a random effect.

Statistical analyses were performed using SAS software version 8.2 (SAS Institute, Cary, NC) in the Medical Computer Programming Department, University Hospital of Montpellier, France.

Sample size was calculated to show a difference of pain intensity at hour 24. In the absence of published data when we started the present study, we estimated that a difference of $25 \mathrm{~mm}$ (estimated common SD of 20) was clinically significant. With a 2-sided significance level of .016 (to take into account a conservative penalty for multiple testing) and a power of $90 \%, 17$ (in each group) patients would be required. We chose to include 60 patients (20 per group).

\section{RESULTS}

Sixty patients underwent nephrectomy using a lumbotomic approach and were randomized preoperatively into the 3 groups: control, EA, and CSSA. Fifty-nine patients successfully completed the study and had a follow-up 3 months postoperatively (Figure 1). The 3 groups were not statistically different for demographic and surgical characteristics, except for age (Table 1). The mean [SD] times (hours) until medically fit for discharge from the CCU were 99 [38] hours in the control group and 64 [24] hours and 63 [13] hours for the EA and CSSA groups, respectively $(P<.001)$. The mean [SD] times (hours) to bowel recovery significantly increased in the control group (86 [19] hours) compared with patients in the EA group (59 [21] hours) and the CSSA group (58 [13] hours) $(P<.001)$; the first time to bedside sitting for more than 2 hours was 83 [13], 51 [15], and 59 [14] hours in the control, EA, and CSSA groups, respectively $(P<.001)$. There was no significant difference in the duration of oxygen supplementation: 68 [46], 46 [28], and 44 [19] hours in the control, EA, and CSSA groups, respectively $(P=.07)$.

\section{Early Postoperative Pain}

During the postoperative 72 hours, analysis of pain intensity indicated a significant difference between groups $(P<.001)$ (Figure 2). Patients in both regional analgesia groups receiving ropivacaine experienced significantly less postoperative pain at rest and during coughing compared with patients in the control group (Figure 2A and B). Pairwise comparisons showed that patients in the EA group had the lowest scores at rest during the first 6 hours $(P<.001)$ and during coughing throughout all postoperative 72 hours $(P<.001)$.

\section{Total Morphine Consumption}

Morphine consumption differed significantly among the groups throughout the postoperative 72 hours (Table 2 ). In the control group, morphine consumption was significantly higher than in the EA and CSSA groups $(P<.001)$. Pairwise comparisons show that in the PACU, patients from the EA group received significantly less morphine than those in the CSSA group. At 24, 48, and 72 hours, the difference in morphine consumption between both ropivacaine groups did not differ significantly.

\section{Area of Hyperalgesia Around the Wound}

The size and type of incision did not differ between the groups (Table 1) but Figure 3 shows that, throughout the first postoperative 72 hours, the median values of total area of hyperalgesia surrounding the surgical incision were lower in both groups of patients receiving ropivacaine compared with the control group. The differences were close to being significant at 48 hours between the EA group $\left(36.4 \mathrm{~cm}^{2}\right)$ and the control group $\left(52 \mathrm{~cm}^{2}\right)(P=.014)$ and at 72 hours among the ropivacaine groups: EA group $\left(40 \mathrm{~cm}^{2}\right)$, CSSA group $\left(39.5 \mathrm{~cm}^{2}\right)$, and the control group $\left(59 \mathrm{~cm}^{2}\right)(P=.002)$. No significant difference was noted between the EA group and the CSSA group throughout the first postoperative 72 hours.

\section{Adverse Events}

The EA and CSSA groups did not differ with regard to adverse effects related to morphine consumption. Nausea or vomiting during the first 72 hours was higher in the control group (85\% and 50\%, respectively) than in the ropivacaine groups (EA group, 30\% and 10\%; CSSA group, 52\% and $21 \%)(P=.012)$. In the control group, $75 \%$ of patients received ondansetron as rescue antiemetic therapy and $30 \%$ received droperidol as second-line therapy compared with $47 \%$ and $21 \%$ in the CSSA group and $15 \%$ and $0 \%$ in the EA group $(P<.001)$. Two hypotensive episodes, 1 cardiac 


\begin{tabular}{|c|c|c|c|c|}
\hline & EA Group $(n=20)$ & CSSA Group $(n=20)$ & Control Group $(n=20)$ & $P$ \\
\hline Age (y) & $66.95(9.83)$ & $57.3(13.79)$ & $56.55(14.51)$ & .02 \\
\hline Sex: male/female & $11 / 9$ & $13 / 7$ & $16 / 4$ & .24 \\
\hline Height $(\mathrm{cm})$ & $169(8)$ & $171(8)$ & $172(7)$ & .63 \\
\hline Weight (kg) & 77.4 (14.89) & $79.4(13.71)$ & $78.65(15.85)$ & .91 \\
\hline Body mass index $\left(\mathrm{kg} / \mathrm{m}^{2} ; \mathrm{SD}\right)$ & 26.88 (4.05) & $27.03(3.46)$ & $26.63(4.69)$ & .95 \\
\hline ASA I/II/III & $3 / 13 / 4$ & $5 / 12 / 3$ & $9 / 8 / 3$ & .33 \\
\hline \multicolumn{5}{|l|}{ Comorbidities } \\
\hline Diabetes mellitus, \% ( $n)$ & $15(3)$ & $20(4)$ & $0(0)$ & .14 \\
\hline Cardiac, \% (n) & $50(10)$ & $60(12)$ & $30(6)$ & .15 \\
\hline Respiratory, \% (n) & $25(5)$ & $30(6)$ & $15(3)$ & .52 \\
\hline Hypertension, \% (n) & $60(12)$ & $50(10)$ & $30(6)$ & .15 \\
\hline \multicolumn{5}{|l|}{ HRQL Parameters } \\
\hline PCS component of HRQL & $44.92(9.52)$ & $46.9(9.71)$ & $49.44(10.22)$ & .35 \\
\hline MCS component of HRQL & $40.25(11.79)$ & $38.71(10.99)$ & $44.47(10.11)$ & .25 \\
\hline \multicolumn{5}{|l|}{ Surgical parameters } \\
\hline Nephrectomy: total/partial & $7 / 13$ & $7 / 13$ & $12 / 8$ & .18 \\
\hline Flank incision/dorsal lumbectomy & $17 / 3$ & $16 / 4$ & $16 / 4$ & 1 \\
\hline Size of incision $(\mathrm{cm})$ & $12.5[12-15]$ & $13[10-15]^{*}$ & 14 [12.5-17.5] & .51 \\
\hline Duration of surgery (min) & 139 (42.31) & 159 (36.69) & 148 (34.99) & .27 \\
\hline Time of surgical retractors use (min) & $104(35.12)$ & $108(26.87)$ & $109(31.61)$ & .87 \\
\hline
\end{tabular}

Values are presented as mean (SD), median [interquartile range], absolute value, or \% frequency (n).

Abbreviations: EA, epidural analgesia; CSSA, continuous surgical site analgesia; SD, standard deviation; ASA, American Society of Anesthesiologists physiological status; HRQL, health-related quality of life; PCS, physical component score; MCS, mental component score.

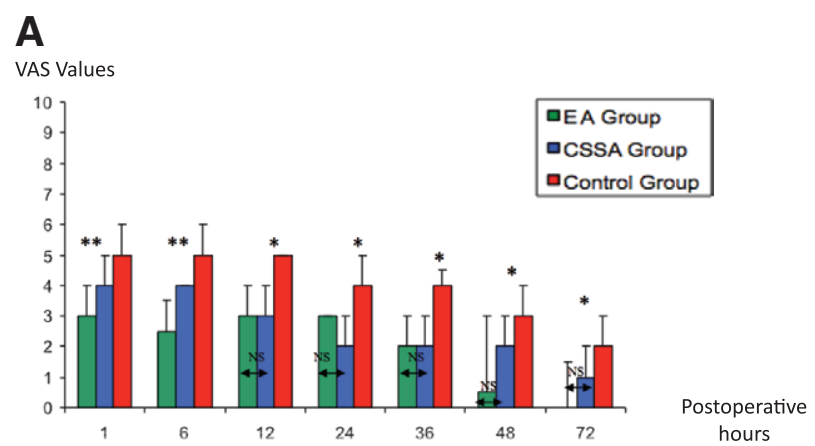

B

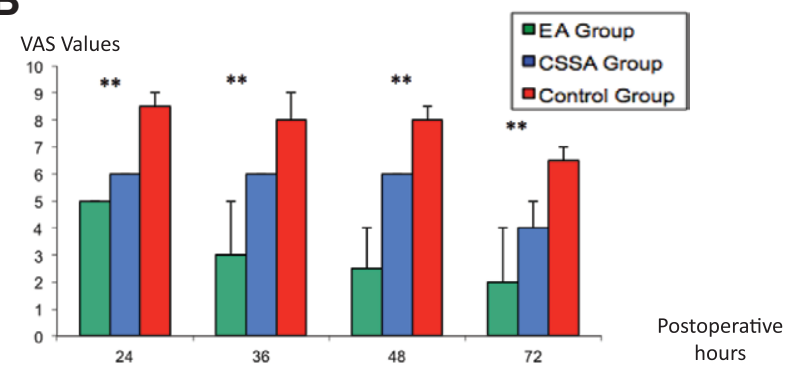

Figure 2. Values of visual analog scale (VAS) pain score at rest (A) and during coughing (B) during the first $72 \mathrm{~h}$ postoperatively. The $P$ values have been calculated using the Kruskal-Wallis test. The boxes represent the median and the bars represent the 75th percentile. ${ }^{*} P<.001$ significant difference; ${ }^{* *} P<.001$ significant difference among all groups. NS indicates nonsignificant difference between the epidural analgesia (EA) group and the continuous surgical site analgesia (CSSA) group (See SM 1 Supplemental material linked to Figure 2, http://links.Iww.com/AA/B558).

arrhythmia, 1 pulmonary edema as well as 2 obstructed catheters, were noted in the EA group $(P=.04$ versus the other 2 groups). One hypotensive episode and 1 catheter were accidentally removed in the CSSA group. One respiratory failure related to pneumonia necessitating a 2-day stay in the intensive care unit and noninvasive ventilation was noted at postoperative day 7 in the control group. We did not have any wound healing or infection in our patients.

\section{Residual Pain and Impact on Quality of Life}

One month after surgery, more than $80 \%$ of patients experienced some residual pain (Figure $4 \mathrm{~A}$ ). The percentage of patients presenting severe pain (necessitating analgesics and/or VAS >4) as well as patients describing hyperalgesia around the wound were lower in the CSSA group (27\% and $21 \%$ ) compared with the EA group ( $44 \%$ and $55 \%$ ) and the control group $(63 \%$ and $55 \%)(P=.02)$. The residual pain values decreased significantly between 1 and 3 months in the CSSA group $(P=0.006)$, whereas decreases of residual pain were not significant in both other groups $(P=0.08)$. As shown in Figure 4B, 3 months after surgery, pain was present for approximately $60 \%$ of patients in both EA and control groups. At 1 month, activities of daily living were affected in $62 \%$ of the patients in the control group versus $30 \%$ in the EA group and 32\% in the CSSA group $(P=.049)$.

At 1 month, the PCS of the SF-36 decreased significantly from preoperative scores in all patients (mean difference: $-10.9[P<.001])$. In EA and control groups at 3 months, mean differences were $-3.11(P=.016)$ and $-12.3(P<.001)$, respectively.

Table 3 shows the preoperative and postoperative scores for the physical and mental subscales according to the patient groups. The analyses showed that preoperative scores were significant variables to explain physical (PF, RP, $\mathrm{BP}$, and general health) and mental components (SF, MH, and RE) after surgery ( $P$ preoperative $<.05)$. Most of the quality-of-life dimensions changed significantly over time (PF, RP, BP, VT, SF, MH, RE; $P$ time < .05), but this evolution differed also between groups $(\mathrm{BP}, \mathrm{MH}, \mathrm{RE} ; P$ time $\times$ group $<.05)$.

$\mathrm{RP}$ and RE scores at 1 month postoperatively were very low for all groups (0), whereas at 3 months postoperatively in EA and control groups, RP scores (0 and 12.5, 


\begin{tabular}{lcccc} 
Table 2. Cumulative Morphine Consumption on a Daily Basis in the $\mathbf{3}$ Groups of Patients \\
EA Group $(\boldsymbol{n}=\mathbf{2 0})$ & CSSA Group $(\boldsymbol{n}=\mathbf{1 9})$ & Control Group $(\boldsymbol{n}=\mathbf{2 0})$ & $10[10-15]$ & \\
PACU & $1[0-7]^{* *}$ & $10[8-14]$ & $32.5[21.5-47]^{*}$ & 4.001 \\
H24 & $10[5-27]$ & $18[12-24]$ & $44[29.5-60.5]^{*}$ & .001 \\
H48 & $20.5[9-41]$ & $24[21-40]$ & $55.5[32.5-72.5]^{*}$ & .002 \\
H72 & $28.5[11-48]$ & $27[24-43]$ & .001 \\
\hline
\end{tabular}

Values are presented as median [interquartile range].

Abbreviations: EA, epidural analgesia; CSSA, continuous surgical site analgesia; PACU, postanesthesia care unit; $\mathrm{H}$, hour.

*Significant difference between other groups.

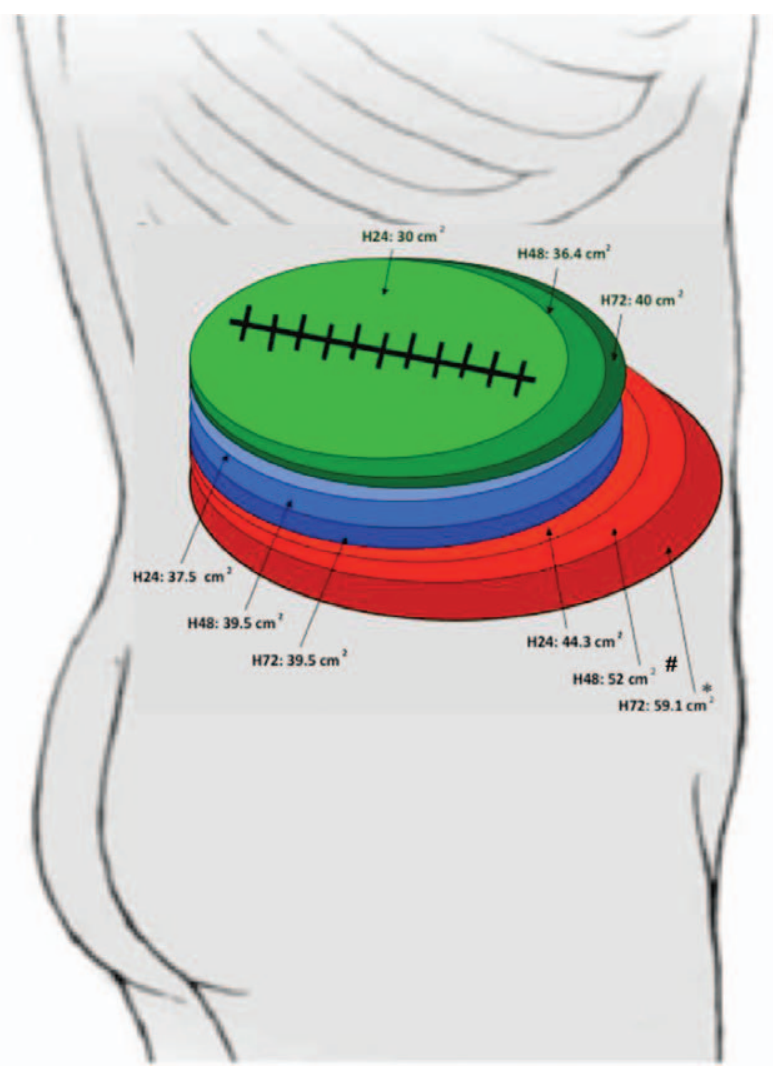

Figure 3. Areas of punctate hyperalgesia around the wound in the three groups of patients at $24 \mathrm{~h}, 48 \mathrm{~h}$, and $72 \mathrm{~h}$ (green: epidural analgesia [EA] group; blue: continuous surgical site analgesia [CSSA] group; red: control group). \# $P<.001$ significant difference between the control and EA groups; ${ }^{* *} P<.001$ significant difference between the control and both ropivacaine groups (See SM 2 Supplemental material linked to Figure 3, http://links.Iww.com/AA/B558).

respectively) and RE scores (16.67 and 33.33, respectively) were still lower than preoperative scores. Scores in the CSSA group recovered for the RP subscale $(75 ; P$ group $=$ .005 and $P$ time $<.001)$ were higher $(66.67)$ than RE preoperative score $(P$ time $\times$ group $<.01)$.

\section{DISCUSSION}

This study is the first prospective randomized comparison among a PCA morphine control group, thoracic epidural, and continuous wound local anesthetic infusions within a multimodal analgesia regimen. Regional analgesia techniques significantly improve postoperative analgesia, reduce postoperative morphine and antiemetic requirements, accelerate CCU stay and recovery of bowel function, and facilitate acute rehabilitation compared with PCA morphine in patients undergoing open nephrectomy.
Furthermore, regional postoperative analgesia decreases the area of wound hyperalgesia. CSSA might significantly reduce the severity of pain and hyperalgesia 1 month after surgery compared with both other groups and optimizes the physical and mental health quality-of-life SF-36 subscales 3 months after surgery.

We report that patients in the EA and CSSA groups experienced significantly less postoperative pain at rest and during coughing compared with patients in the PCA morphine group. CSSA is an alternative to EA for postoperative analgesia after open renal surgery. ${ }^{12-14,18}$ Forastière et $\mathrm{al}^{12}$ reported reduced VAS pain scores with CSSA compared with PCA morphine within the first postoperative 48 hours. For open colorectal surgery, ${ }^{11}$ CSSA using $10 \mathrm{~mL} / \mathrm{h}$ of $0.2 \%$ ropivacaine significantly reduced pain scores. Patients in the EA group reported significantly lower pain scores at rest during the first 6 hours postoperatively and for 72 hours during coughing. EA could be considered as a prerequisite for optimal postoperative analgesia after open nephrectomy. ${ }^{5,6,19}$ After open colorectal surgery and in agreement with our results, Jouve et $\mathrm{al}^{15}$ reported that EA (basal bolus regimen of ropivacaine $0.2 \%$ and $0.25 \mu \mathrm{g} / \mathrm{mL}$ sufentanil) provided lower pain scores at rest than CSSA in the first 8 hours and during 72 hours postoperatively for dynamic pain. Conversely, Bertoglio et $\mathrm{al}^{16}$ reported that preperitoneal CSSA provided lower pain scores compared with EA (continuous infusion of $0.2 \%$ ropivacaine) at 48 and 72 hours. Finally, a recent meta-analysis ${ }^{20}$ reported that the use of local anesthetic wound infiltration gave pain scores comparable with those obtained with EA after abdominal surgery.

In the control group, morphine consumption was significantly higher than in the EA and CSSA groups. It has been well demonstrated that the use of EA or CSSA compared with PCA morphine revealed significantly less use of rescue opioid for patients administered ropivacaine. ${ }^{11,12,18,21}$ Forastière et $\mathrm{al}^{12}$ reported significantly decreased mean total morphine consumption over the first postoperative 48 hours in the CSSA group after open nephrectomy (11.5 [0.27] $\mathrm{mg}$ ) compared with the PCA morphine group (21.8 [0.37] mg). Our results showed higher consumption of morphine in the CSSA and control groups compared with Forastière et al's study ${ }^{12}$ during the first 48 hours postoperatively despite the use of a multimodal analgesia regimen. The differences may be the result of the preoperative wound infiltration of $10 \mathrm{~mL}$ of $1 \%$ ropivacaine, mean duration of surgery ( 78 minutes vs 159 minutes), and systematic use of ketamine in the other study.

Adequate pain management minimizes morphine consumption, increases patient mobility, decreases hospital stay, and optimizes patient outcome. ${ }^{22,23}$ In our study, both 

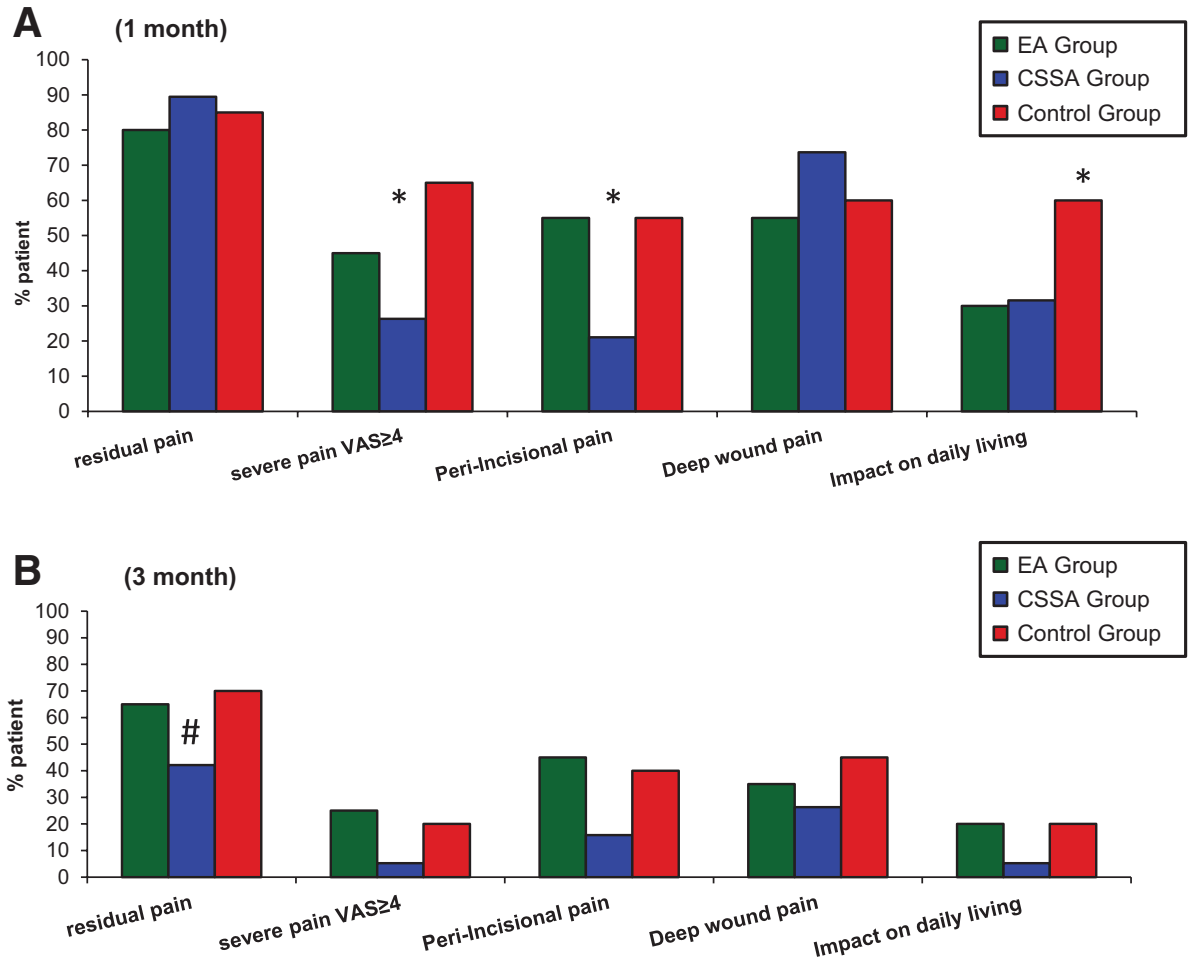

Figure 4. Chronic pain characteristics in the three groups of patients 1 and 3 mo after surgery. ${ }^{*} P<.001$ significant difference between the continuous surgical site analgesia (CSSA) group or the control group and the other two groups at 1 month for the selected characteristics. $\# P=.006$ significant difference in residual pain evolution in the CSSA group compared with both other groups. regional analgesia groups have significantly accelerated return of bowel function, chairside sitting, and discharge from the CCU compared with the control group. Nausea and vomiting during the first 72 hours were higher in the PCA morphine group. Previous data ${ }^{12,18}$ support our hypothesis. In Forastière et al's study, ${ }_{1}^{12}$ the times to bowel recovery and discharge as well as the incidence of nausea and vomiting decreased in the CSSA group compared with PCA morphine group. Focusing on early postoperative outcome parameters and adverse events, CSSA is a reasonable alternative to EA after lumbotomy.

Peripheral nociceptive input from the surgical site promotes incisional pain, hypersensitivity, ${ }^{24}$ and central sensitization ${ }^{25-28}$ leading to persistent pain in some cases. We showed that in the first postoperative 72 hours, the total area of hyperalgesia surrounding the surgical incision was lower in both groups of patients receiving ropivacaine compared with the control group. After major gastrointestinal surgery, ${ }^{9}$ the area of hyperalgesia around a laparotomy incision has been reported significantly higher (mean [SD] 155 [30] $\mathrm{cm}^{2}$ at 72 hours) in a group of patients receiving a systemic postoperative analgesia regimen compared with 3 other groups of patients scheduled for intraoperative, perioperative, or postoperative EA (mean [SD] 20 [10] $\mathrm{cm}^{2}$ at 72 hours). The only study ${ }^{29}$ on CSSA ( $5 \mathrm{~mL} / \mathrm{h}$ of ropivacaine $2 \%$ ) compared with placebo plus $75 \mathrm{mg}$ IV diclofenac in both groups reported that the area of punctate hyperalgesia around the cesarean delivery incision did not differ. We demonstrated that the use of CSSA or EA decreased the hyperalgesia area compared with systemic opiates. The difference between both studies using a continuous wound infusion is probably related to the use of IV diclofenac in both groups in the previous study.
We report that CSSA is superior for reducing central sensitization and limiting the risk of residual pain after lumbotomy compared with both other groups. At 1 month, the percentage of patients reporting severe pain and/or hyperalgesia was significantly lower in the CSSA group compared with the EA and control groups. Lavand'homme et $\mathrm{al}^{9}$ reported that patients receiving EA only in the postoperative period did not differ in terms of residual pain at 1 month compared with a control group. The studies using CSSA compared with systemic analgesia did not report any difference in residual pain at 1 and 3 months. Some authors reported no pain ${ }^{12,15,16,30}$ or a $5 \%$ incidence. ${ }^{11}$ In contrast to our study, the prospective quantification of chronic pain (type of pain, measured pain level, location) was not specifically evaluated in other studies. Three months after surgery, some preoperative scores for the PCS and MCS subscales had not recovered in the EA and control groups but were significantly higher in the CSSA group (Table 3). After open nephrectomy using an EA regimen, Gerbershagen et $\mathrm{al}^{8}$ reported that psychologic and psychosocial status had worsened in patients developing chronic pain within 3 months postoperatively. Levy et $\mathrm{al}^{31}$ did not report any difference in all components (MCS and PCS) of the SF-36 questionnaire between EA and PCA morphine groups 1 month after laparoscopic colorectal surgery. Conversely, Carli et $\mathrm{al}^{10}$ noted that after open colon surgery, the EA group experienced an improvement in all SF-36 subscales 6 weeks after surgery compared with the PCA morphine group. Our study is the first to analyze HRQL related to postoperative wound infusion. We report that CSSA resulted in improved scores for the physical and mental subscales of the SF-36 questionnaire compared with EA and PCA morphine 3 months after open nephrectomy.

Our study has several limitations. From a statistical point of view, the sample size was calculated without a 


\begin{tabular}{|c|c|c|c|c|}
\hline & EA Group $(n=20)$ & CSSA Group $(n=19)$ & Control Group $(n=20)$ & $\boldsymbol{P}$ \\
\hline \multicolumn{5}{|c|}{ Physical component summary } \\
\hline Preoperative & $44.6[35.8 ; 51.4]$ & $46.5[38.8 ; 53.3]$ & $52.2[45.3 ; 57.3]$ & $P$ preoperative $<.001$ \\
\hline $1 \mathrm{mo}$ & $36.8[31.7 ; 39.9]$ & $39.1[30.8 ; 44.1]$ & $36.4[33.3 ; 40]$ & $P$ group $=.52$ \\
\hline $3 \mathrm{mo}$ & $39.6[35.1 ; 48.9]$ & $47[41.5 ; 54.3]$ & 43. [38.6;49.8] & $\begin{array}{l}P \text { time }<.001 \\
P \text { time } \times \text { group }=.44\end{array}$ \\
\hline \multicolumn{5}{|c|}{ Physical functioning } \\
\hline Preoperative & $60[42.5 ; 92.5]$ & 75 [55; 95] & $92.5[80 ; 100]$ & $P$ preoperative $=.006$ \\
\hline $1 \mathrm{mo}$ & $40[30 ; 67.5]$ & $60[30 ; 70]$ & $60[47.5 ; 67.5]$ & $P$ group $=.26$ \\
\hline $3 \mathrm{mo}$ & $62.5[40 ; 72.5]$ & $80[55 ; 95]$ & $77.5[67.5 ; 90]$ & $\begin{array}{l}P \text { time }<.0001 \\
P \text { time } \times \text { group }=.90\end{array}$ \\
\hline \multicolumn{5}{|l|}{ Role physical } \\
\hline Preoperative & $25[0-100]$ & $75[0-100]$ & $87.5[0-100]$ & $P$ preoperative $=.0036$ \\
\hline $1 \mathrm{mo}$ & $0[0-0]$ & $0[0-50]$ & $0[0-0]$ & $P$ group $=.0056$ \\
\hline $3 \mathrm{mo}$ & $0[0-50]$ & $75[0-100]^{*}$ & $12.5[0-50]$ & $\begin{array}{l}P \text { time }<.0001 \\
P \text { time } \times \text { group }=.13\end{array}$ \\
\hline \multicolumn{5}{|l|}{ Bodily pain } \\
\hline Preoperative & 77 [62-100] & 64 [51-100] & 100 [62-100] & $P$ preoperative $=.03$ \\
\hline $1 \mathrm{mo}$ & 62 [42-74] & 52 [31-62] & 41 [36-57] & $P$ group $=.52$ \\
\hline $3 \mathrm{mo}$ & 74 [57-84] & 80 [52-100] & 69 [62-74] & $\begin{array}{l}P \text { time }<.0001 \\
P \text { time } \times \text { group }=.04\end{array}$ \\
\hline \multicolumn{5}{|l|}{ General Health } \\
\hline Preoperative & 57 [47-63.5] & 55 [47-62] & 67 [44.5-82] & $P$ preoperative $<.0001$ \\
\hline $1 \mathrm{mo}$ & 57 [37-67] & $47[42-72]$ & 62 [46-82] & $P$ group $=.99$ \\
\hline $3 \mathrm{mo}$ & $57[38.5-67]$ & 52 [40-72] & $64.5[49.5-82]$ & $\begin{array}{l}P \text { time }=.2 \\
P \text { time } \times \text { group }=.47\end{array}$ \\
\hline \multicolumn{5}{|c|}{ Mental component summary } \\
\hline Preoperative & $41.3[33 ; 49.7]$ & $36.1[27.8 ; 46.4]$ & $43[37.6 ; 55.3]$ & $P$ preoperative $=.008$ \\
\hline $1 \mathrm{mo}$ & $38.7[32.6 ; 47.6]$ & 37 [32.5;49.9] & $41.6[33.1 ; 46.9]$ & $P$ group $=.64$ \\
\hline $3 \mathrm{mo}$ & $37.2[33.8 ; 42.5]$ & $48.8[34 ; 51.8]$ & $45.3[42.6 ; 54.4]$ & $\begin{array}{l}P \text { time }=.02 \\
P \text { time } \times \text { group }=.03\end{array}$ \\
\hline \multicolumn{5}{|l|}{ Vitality } \\
\hline Preoperative & 50 [35-65] & 45 [30-65] & $62.5[40-75]$ & \\
\hline $1 \mathrm{mo}$ & $47.5[35-57.5]$ & $40[25-60]$ & $47.5[37.5-60]$ & $P$ preoperative $=.15$ \\
\hline $3 \mathrm{mo}$ & 50 [35-55] & $55[45-70]$ & 55 [52.5-67.5] & $\begin{array}{l}P \text { group }=.06 \\
P \text { time }<.0001 \\
P \text { time } \times \text { group }=.0055\end{array}$ \\
\hline \multicolumn{5}{|c|}{ Social functioning } \\
\hline Preoperative & $56.25[43.8-75]$ & 62.5 [37.5-87.5] & 68.75 [62.5-87.5] & $\begin{array}{l}P \text { preoperative }<.0001 \\
P \text { group }=.26\end{array}$ \\
\hline $1 \mathrm{mo}$ & $50[31.25-75]$ & $62.5[50-75]$ & $62.5[37.5-75]$ & $\begin{array}{l}P \text { time }<.001 \\
P \text { time } \times \text { group }=.62\end{array}$ \\
\hline $\begin{array}{l}3 \mathrm{mo} \\
\text { Mental health }\end{array}$ & $56.25[50-75]$ & 75 [50-87.5] & 75 [56.25-87.5] & \\
\hline Preoperative & $54[44-68]$ & $48[40-64]$ & $64[48-82]$ & $P$ preoperative $=.0017$ \\
\hline $1 \mathrm{mo}$ & $60[48-70]$ & 56 [44-68] & $66[48-78]$ & $P$ group $=.74$ \\
\hline $3 \mathrm{mo}$ & 54 [46-64] & $68[52-72]$ & 70 [56-84] & $\begin{array}{l}P \text { time }=.03 \\
P \text { time } \times \text { group }=.0031\end{array}$ \\
\hline \multicolumn{5}{|l|}{ Role emotional } \\
\hline Preoperative & 50 [0-100] & 33.33 [0-100] & 83.33 [16.7-100] & $P$ preoperative $=.02$ \\
\hline $1 \mathrm{mo}$ & $0[0-50]$ & 0 [0-66.67] & 0 [0-33.3] & $\begin{array}{l}P \text { group }=.47 \\
P \text { time }<.0001\end{array}$ \\
\hline $3 \mathrm{mo}$ & $16.67[0-100]$ & $66.67[0-100]$ & 33.33 [16.67-100] & $P$ time $\times$ group $=.23$ \\
\hline
\end{tabular}

Abbreviations: EA, epidural analgesia; CSSA, continuous surgical site analgesia.

pilot study, which could have given valuable information concerning the success rate of different groups. Therefore, we made a speculation, which led to 3 groups of 20 patients each. The samples are small and the possibility of a type II error exists. The study was not a double-blind trial because we were not able to obtain permission from our ethical committee. The committee considered it unethical and dangerous for the patients to have single-sham (regional groups) or double-sham (control group) catheter lines with the patient being blinded about the analgesia regimen.
However, all parameters were recorded by 2 research physicians not involved in the primary phase of the study and intraoperative patient care. The use of postoperative EA (rather than intra- and postoperative EA) can be challenged. ${ }^{32}$ Lavand'homme et $\mathrm{al}^{9}$ reported the same results for postoperative VAS scores, areas of hyperalgesia, and residual pain necessitating treatment in 3 groups of patients receiving perioperative and only intra- or postoperative EA after colon surgery. All groups were different from the systemic analgesia group. Barreveld et $\mathrm{al}^{33}$ reported in a recent 
systematic review that a significant antihyperalgesic effect of a regional analgesia procedure occurs when the local anesthetic is present during the early postoperative period and that its presence during surgery is not crucial for that effect. However, we should note that their test for hyperalgesia at 1 and 3 months was with a questionnaire unlike our testing, which was done with a monofilament.

In conclusion, our results show that CSSA and EA significantly improve postoperative analgesia, reduce postoperative morphine consumption, decrease the area of wound hyperalgesia, and accelerate patient rehabilitation in an enhanced recovery program of patients undergoing open nephrectomy. CSSA significantly reduces the severity of residual pain 1 month after surgery and optimizes HRQL parameters 3 months after surgery compared with the EA and control groups.

\section{DISCLOSURES}

Name: Xavier Capdevila, MD, PhD.

Contributor: This author helped design the study, conduct the study, analyze the data, and write the manuscript.

Name: Sebastien Moulard, MD.

Contributor: This author helped design the study, conduct the study, analyze the data, and write the manuscript.

Name: Christian Plasse, MD.

Contributor: This author helped conduct the study.

Name: Jean-Luc Peshaud, MD.

Contributor: This author helped conduct the study.

Name: Nicolas Molinari, PhD.

Contributor: This author helped design the study and analyze the data.

Name: Christophe Dadure, MD, $\mathrm{PhD}$.

Contributor: This author helped conduct the study and write the manuscript.

Name: Sophie Bringuier, PharmD, PhD.

Contributor: This author helped design the study, analyze the data, and write the manuscript.

This manuscript was handled by: Honorio T. Benzon, MD.

\section{REFERENCES}

1. Couapel JP, Bensalah K, Bernhard JC, et al. Is there a volumeoutcome relationship for partial nephrectomy? World J Urol. 2014;32:1323-1329.

2. Sharma V, Margreiter M. Partial nephrectomy: is there still a need for open surgery? Curr Urol Rep. 2013;14:1-4.

3. Gerbershagen HJ, Aduckathil S, van Wijck AJ, Peelen LM, Kalkman CJ, Meissner W. Pain intensity on the first day after surgery: a prospective cohort study comparing 179 surgical procedures. Anesthesiology. 2013;118:934-44.

4. Gerbershagen HJ, Pogatzki-Zahn E, Aduckathil S, et al. Procedure-specific risk factor analysis for the development of severe postoperative pain. Anesthesiology. 2014;120:1237-1245.

5. Moraca RJ, Sheldon DG, Thirlby RC. The role of epidural anesthesia and analgesia in surgical practice. Ann Surg. 2003;238:663-673.

6. Wuethrich PY, Metzger T, Mordasini L, Kessler TM, Curatolo M, Burkhard FC. Influence of epidural mixture and surgery on bladder function after open renal surgery: a randomized clinical trial. Anesthesiology. 2013;118:70-77.

7. Niraj G, Kelkar A, Jeyapalan I, et al. Comparison of analgesic efficacy of subcostal transversus abdominis plane blocks with epidural analgesia following upper abdominal surgery. Anaesthesia. 2011;66:465-471.

8. Gerbershagen HJ, Dagtekin O, Rothe T, et al. Risk factors for acute and chronic postoperative pain in patients with benign and malignant renal disease after nephrectomy. Eur J Pain. 2009;13:853-860.

9. Lavand'homme $P$, De Kock $M$, Waterloos H. Intraoperative epidural analgesia combined with ketamine provides effective preventive analgesia in patients undergoing major digestive surgery. Anesthesiology. 2005;103:813-820.
10. Carli F, Mayo N, Klubien K, Schricker T, Trudel J, Belliveau P. Epidural analgesia enhances functional exercise capacity and health-related quality of life after colonic surgery: results of a randomized trial. Anesthesiology. 2002;97:540-549.

11. Beaussier M, El'Ayoubi H, Schiffer E, et al. Continuous preperitoneal infusion of ropivacaine provides effective analgesia and accelerates recovery after colorectal surgery: a randomized, double-blind, placebo-controlled study. Anesthesiology. 2007;107:461-468.

12. Forastiere E, Sofra M, Giannarelli D, Fabrizi L, Simone G. Effectiveness of continuous wound infusion of $0.5 \%$ ropivacaine by On-Q pain relief system for postoperative pain management after open nephrectomy. Br J Anaesth. 2008;101:841-847.

13. Panaro F, Gheza F, Piardi T, et al. Continuous infusion of local anesthesia after living donor nephrectomy: a comparative analysis. Transplant Proc. 2011;43:985-987.

14. Sorbello M, Paratore A, Morello G, et al. Wound levobupivacaine continuous infusion for postoperative analgesia in living kidney donors: case-control study. Transplant Proc. 2009;41:1128-1131.

15. Jouve P, Bazin JE, Petit A, et al. Epidural versus continuous preperitoneal analgesia during fast-track open colorectal surgery: a randomized controlled trial. Anesthesiology. 2013;118:622-630.

16. Bertoglio S, Fabiani F, Negri PD, et al. The postoperative analgesic efficacy of preperitoneal continuous wound infusion compared to epidural continuous infusion with local anesthetics after colorectal cancer surgery: a randomized controlled multicenter study. Anesth Analg. 2012;115:1442-1450.

17. Ware JE Jr, Sherbourne CD. The MOS 36-item short-form health survey (SF-36). I. Conceptual framework and item selection. Med Care. 1992;30:473-483.

18. Biglarnia AR, Tufveson G, Lorant T, Lennmyr F, Wadström J. Efficacy and safety of continuous local infusion of ropivacaine after retroperitoneoscopic live donor nephrectomy. Am J Transplant. 2011;11:93-100.

19. Suarez-Sanchez L, Perales-Caldera E, Pelaez-Luna MC, BernalFlores R. Postoperative outcome of open donor nephrectomy under epidural analgesia: a descriptive analysis. Transplant Proc. 2006;38:877-881.

20. Ventham NT, Hughes M, O'Neill S, Johns N, Brady RR, Wigmore SJ. Systematic review and meta-analysis of continuous local anaesthetic wound infiltration versus epidural analgesia for postoperative pain following abdominal surgery. $\mathrm{Br} \mathrm{J}$ Surg. 2013;100:1280-1289.

21. Raines S, Hedlund C, Franzon M, Lillieborg S, Kelleher G, Ahlén K. Ropivacaine for continuous wound infusion for postoperative pain management: a systematic review and meta-analysis of randomized controlled trials. Eur Surg Res. 2014;53:43-60.

22. Rigg JR, Jamrozik K, Myles PS, et al; MASTER Anaethesia Trial Study Group. Epidural anaesthesia and analgesia and outcome of major surgery: a randomised trial. Lancet. 2002;359:1276-1282.

23. Liu SS, Carpenter RL, Mackey DC, et al. Effects of perioperative analgesic technique on rate of recovery after colon surgery. Anesthesiology. 1995;83:757-765.

24. Brennan TJ. Frontiers in translational research: the etiology of incisional and postoperative pain. Anesthesiology. 2002;97:535-537.

25. Zahn PK, Brennan TJ. Primary and secondary hyperalgesia in a rat model for human postoperative pain. Anesthesiology. 1999;90:863-872.

26. Obata H, Eisenach JC, Hussain H, Bynum T, Vincler M. Spinal glial activation contributes to postoperative mechanical hypersensitivity in the rat. J Pain. 2006;7:816-822.

27. Peles S, Miranda A, Shaker R, Sengupta JN. Acute nociceptive somatic stimulus sensitizes neurones in the spinal cord to colonic distension in the rat. J Physiol. 2004;560:291-302.

28. Eisenach JC. Preventing chronic pain after surgery: who, how, and when? Reg Anesth Pain Med. 2006;31:1-3.

29. Lavand'homme PM, Roelants F, Waterloos H, De Kock MF. Postoperative analgesic effects of continuous wound infiltration with diclofenac after elective cesarean delivery. Anesthesiology. 2007;106:1220-1225. 
30. Fassoulaki A, Chassiakos D, Melemeni A. Intermittent epidural vs continuous wound infusion of ropivacaine for acute and chronic pain control after hysterectomy or myomectomy: a randomized controlled trial. Pain Med. 2014;15:1603-1608.

31. Levy BF, Scott MJ, Fawcett W, Fry C, Rockall TA. Randomized clinical trial of epidural, spinal or patient-controlled analgesia for patients undergoing laparoscopic colorectal surgery. $\mathrm{Br} J$ Surg. 2011;98:1068-1078.
32. Lassen K, Soop M, Nygren J, et al; Enhanced Recovery After Surgery (ERAS) Group. Consensus review of optimal perioperative care in colorectal surgery: enhanced recovery after surgery (ERAS) group recommendations. Arch Surg. 2009;144:961-969.

33. Barreveld A, Witte J, Chahal H, Durieux ME, Strichartz G. Preventive analgesia by local anesthetics: the reduction of postoperative pain by peripheral nerve blocks and intravenous drugs. Anesth Analg. 2013;116:1141-1161. 